\title{
Controle biológico em sistema orgânico de produção por agricultores da cidade de Maringá (Paraná, Brasil)
}

\author{
Biological control on organic agriculture by growers of the city of maringá (Paraná, \\ Brazil) \\ Francieli das Chagas, Julio Cesar Polonio , Maria Claudia Colla Ruvolo-Takasusuki, \\ João Alencar Pamphile e Helio Conte
}

\author{
Universidade Estadual de Maringá/Programa de Pós-Graduação em Biotecnologia Ambiental \\ franci_chagas@hotmail.com; julioc_polonio@hotmail.com; mccrtakasusuki@uem.br; \\ prof.pamphile@gmail.com; hconte@uem.br
}

\begin{abstract}
Resumo
O aumento contínuo da população mundial e o uso intensificado de agrotóxicos na agricultura tornaram os problemas ambientais evidentes, levando a produção agrícola a desenvolver práticas alternativas que favoreçam a sustentabilidade ambiental. O uso de técnicas de controle biológico para redução de pragas agrícolas tem sido crescente, permitindo a substituição de inseticidas convencionais por inimigos naturais diversificados como insetos, vírus, fungos, bactérias, extratos vegetais, dentre outros. Foi realizada uma análise quantitativadescritiva, utilizando-se um questionário como forma de entrevista pessoal em amostras aleatórias com agricultores do sistema orgânico de produção da cidade de Maringá-PR, Brasil. O objetivo foi quantificar o conhecimento, utilização e viabilidade econômica do uso de controladores biológicos, promovendo a ampliação da prática e desenvolvimento de novos controladores que substituam ou reduzam o uso de defensivos químicos. Os dados mostraram que todos os produtores entrevistados possuem o conhecimento e já utilizaram métodos alternativos de controle de pragas, parte considera a prática de grande importância ambiental, porém ainda são necessários maiores estudos sobre a entomofauna regional para consolidação e melhor eficácia do biocontrole.
\end{abstract}

Palavras-chave: Agentes Controladores, Manejo Integrado de Pragas, Entomopatógenos, Combinação biológico-química, Extratos Vegetais.

\begin{abstract}
The continuous growth of world population and the increased use of pesticides became obvious environmental problems, leading to agricultural production to develop alternative practices that promote environmental sustainability. The use of biological control techniques to reduce agricultural pests have been increasing, enabling the substitution of conventional insecticides by diversified natural enemies such as insects, viruses, fungi, bacteria, plant extracts, and others. A quantitative-descriptive analysis was carried using a questionnaire as mean to personal interview of organic growers in the city of Maringa, state of Paraná, Brazil was held. The aim was quantify about the knowledge of biological controllers by this growers, the use, and economic viability of these, aiming to promote the expansion of the practice and development of new controllers to replace or reduce the use of chemicals. The results show that all growers have the knowledge and have used alternative methods of biocontrol, some of them consider the practice of great environmental importance, but is still required further studies of the regional entomofauna to better effectiveness of the biocontrol.
\end{abstract}

Keywords: Control agents, Integrated pest management, Entomopathogens, Bio-chemicals combinations, Plant Extracts. 


\section{Introdução}

O uso intensificado de agrotóxicos na agricultura tem contribuído para o detrimento da saúde humana, ambiental, e da biodiversidade (LIMA; ROCHA, 2012). Esses efeitos sobre o ambiente tornaram o uso do controle biológico natural ou aplicado uma realidade (PARRA, 1992).

O novo direcionamento da produção agrícola é desenvolver práticas de menor impacto ambiental no combate de pragas, onde por meio de métodos alternativos substitui ou reduz-se a utilização de químicos com efeitos negativos à saúde humana e meio ambiente, favorecendo o uso sustentável da biodiversidade (LACEY et al., 2001; SILVA et al., 2010).

A ocorrência natural de controladores é definida pela regulação da população dentro de certos limites e em períodos de tempos, por combinações de fatores naturais (DEBACH; ROSEN, 1991).

Uma alternativa para o controle de pragas agrícolas é a introdução de agentes controladores naturais em um determinado ambiente. Neste quesito, pode se utilizar o controle biológico clássico, que consiste na importação e colonização de parasitoides ou predadores, visando o controle de pragas exóticas, ou eventualmente nativas; e o controle biológico aplicado, a partir da liberação de parasitoides ou predadores após a sua produção massal em laboratório, visando à redução rápida da população praga para um nível de equilíbrio. Tal metodologia possui maior aceitação por sua ação rápida, semelhante a inseticidas convencionais (GALLO et al., 2002).

No Brasil, os programas de controle biológico são comparáveis aos melhores do mundo, podendo citar os agentes controladores: Cotesia flavipes (Hymenoptera:

Braconidae), Metagonistylum minense (Diptera: Tachinidae), Paratheresia claripalpis (Diptera: Tachinidae), Trichogramma sp. (Hymenoptera: Trichogrammatidae) Baculovirus anticarsia (Baculoviridae), além de outros projetos menores envolvendo o uso de parasitoides, predadores ou patógenos (vírus, bactérias e fungos). Porém, existem grandes problemas relacionados com o desenvolvimento de técnicas de controle biológico devido a limitações de recursos para pesquisas e pouca literatura sobre o tema, além do pequeno número de pesquisadores envolvidos (PARRA, 1992; PARRA, 2014).

A aplicação de extratos vegetais como inseticida também é uma prática de controle biológico utilizada desde os ancestrais Romanos até os dias atuais, destacando-se mais de duas mil espécies vegetais por suas propriedades pesticidas (CHAGAS, 2004). Uma das técnicas utilizadas até a metade do século XIX inclui óleos essenciais como inseticidas e fungicidas no controle de pragas e doenças agrícolas, dentre estes aqueles à base de Chrysanthemum roseum Adams (Asterales: Asteraceae) e $C$. coccineum Jacquin (Asterales: Asteraceae) como fontes de piretro; Derris spp. (Fabales: Fabaceae) e Lonchocarpus spp. (Fabales: Fabaceae) como fontes de rotenona; e Nicotiana 
como fontes de nicotina (MORAIS, 2009).

Embora de maneira informal, agricultores utilizam controladores na cidade de Maringá-PR, não existem dados quantificados e informações sobre tal prática. Assim, a realização do presente estudo tem por objetivo verificar a atual situação do uso de controladores biológicos, do conhecimento, da aplicação das técnicas e viabilidade econômica, na perspectiva de agricultores em sistemas orgânicos de produção na cidade de Maringá PR. Tais informações poderão permitir a ampliação da prática para embasar o desenvolvimento de novas técnicas aplicáveis.

\section{Material e métodos}

A presente pesquisa foi realizada no período de Abril a Maio de 2015, na cidade de Maringá - PR, Brasil $\left(23^{\circ} 24^{\prime} 19^{\prime \prime S} 51^{\circ} 56^{\prime} 29^{\prime \prime} \mathrm{W}\right)$ a partir de uma análise quantitativa-descritiva utilizando questionário (disponível como material suplementar) como forma de entrevista pessoal em amostras aleatórias de pequenos agricultores do sistema orgânico de produção.

O número amostral de agricultores participantes do questionário foi selecionado a partir de dados fornecidos pela EMATER (Empresa Brasileira de Extensão Rural, Maringá-PR) e POMAR (Associação dos Produtores Orgânicos de Maringá), na qual foram selecionados apenas os agricultores com área inferior a 50 hectares no município de Maringá - PR e que possuíam o certificado da
POMAR de produtores orgânicos rurais. A faixa etária foi definida entre 21 a 70 anos, com ao menos o ensino fundamental completo.

O contato com os participantes selecionados para o estudo foi realizado pessoalmente após a apresentação do Termo de consentimento livre e esclarecido (TCLE) (disponível como material suplementar), no qual foi previamente aprovado pelo Comitê de Ética em Pesquisa com Seres Humanos da Universidade Estadual de Maringá (COPEP-UEM) com registro na Plataforma Brasil (CAAE: 39678914.0.0000.0104). A aplicação do questionário foi durante a presença dos agricultores nas feiras-livres de produtos hortifrutigranjeiros, "Feira do Produtor da Zona 7", realizadas semanalmente no centro urbano da cidade de Maringá.

A tabulação dos dados e criação dos gráficos foi realizada utilizado o software Microsoft ${ }^{\circledR}$ Excel versão 2010.

\section{Resultados e Discussão}

Para a realização da pesquisa, foram entrevistados 11 agricultores. Todos apontaram ter conhecimento sobre "o que seria o controle biológico de pragas" e que já utilizaram alguma forma de biocontrole. Os participantes afirmaram que os resultados esperados com o uso de biocontroladores foram atingidos de acordo com as informações fornecidas pelo fabricante (quando adquirido comercialmente).

Dez agricultores relataram já terem recebido a sugestão para o uso e dentre estes, nove receberam treinamento e consideram o uso de 
biocontroladores economicamente viável, com resultados satisfatórios (mesmo com a ocorrência de perdas) e de grande importância ambiental.

Franceschini et al. (2001), consideram o controle biológico uma alternativa para o combate de pragas e patógenos quando comparada ao controle químico, especialmente relacionado ao impacto ambiental, custo, especificidade e desenvolvimento de resistência. Entretanto, nem todas as espécies invasoras são passíveis ao uso de controladores, existindo a necessidade de um agente de especificidade adequada. Em casos onde várias espécies invasivas requerem controle, o controle biológico de um invasor por si só pode não ser suficiente para alcançar a restauração ecológica de uma comunidade (VAN DRIESCHE et al., 2010).

As principais plantas cultivadas pelos agricultores orgânicos da cidade estão demonstradas na Figura 1. Dentre estas, destacam-se as hortaliças e outras culturas como tomate, morango, banana, mandioca, soja, Citrus em geral, sendo produzidas sem o uso de pesticidas químicos. Foram citadas com menor frequência culturas de milho, feijão, algumas frutas como caqui e ameixa, eucalipto entre outros.

A especificidade de algumas pragas em determinadas culturas pode estar correlacionada ao maior índice de infestação em regiões específicas, ao clima e espécies cultivadas. As pragas citadas com maior ocorrência foram os pulgões (26\% dos entrevistados), percevejos $(17 \%)$, lagartas, principalmente Helicoverpa armigera
(Lepidoptera: Noctuidae) (15\%), Moscabranca, citada a espécie Bemisia tabaci (Hemiptera: Aleyrodidae) (15\%), a Vaquinha, podendo ser citada as do gênero Diabrotica spp. (Coleoptera: Chrysomelidae) (12\%) e formigas (Hymenoptera: Formicidae) 6\% (Figura 2).

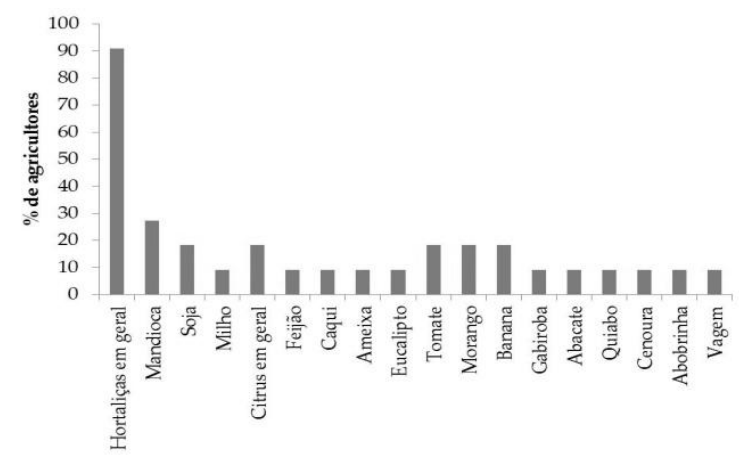

Figura 1 - Principais culturas em sistema orgânico de produção, cultivadas pelos agricultores da cidade de Maringá-PR

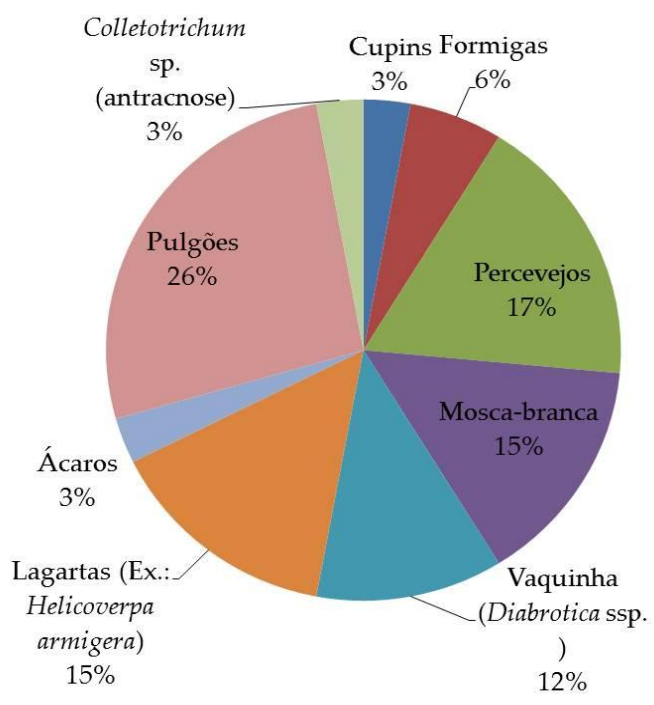

Figura 2 - Principais pragas agrícolas que afetam as culturas orgânicas dos agricultores

Os defensivos agrícolas apresentam uma alta e rápida eficiência 
propiciando aumento na produtividade. Porém, sua eficácia exige a repetição das aplicações, representando um custo elevado ao produtor, além de acarretar efeitos negativos sobre o solo, a vegetação, as águas, aos animais e ao homem. Determinadas substâncias químicas levam décadas para se degradarem, elevando a concentração dessas substâncias na cadeia alimentar e ocasionando a seleção de mutantes resistentes, resultantes da pressão seletiva (FRANCESCHINI et al., 2001).

Com o presente estudo foi constatado o uso de diversos controles alternativos para pragas (Figura 3), incluindo extratos de plantas, caldas sulfocáusticas e microrganismos entomopatogênicos como os fungos Metarhizium anisopliae (Metschn.) Sorokīn (Hypocreales: Clavicipitaceae) e Beauveria bassiana (Bals.-Criv.) Vuill. (Hypocreales: Cordycipitaceae), o micoparasita Trichoderma sp. (Hypocreales: Hypocreaceae) e a bactéria Bacillus thuringiensis (Bacillales: Bacillaceae). Também foram citadas práticas homeopáticas e a inserção de alguns insetos controladores como ácaros e joaninhas, além da manutenção da área verde da propriedade (manutenção da biodiversidade).

O uso de substâncias naturais provenientes de plantas medicinais, aromáticas e condimentares, é uma alternativa para o controle das doenças de plantas, além da fácil obtenção, baixo custo e sem toxidez residual (MORAIS, 2009).

De acordo com Mora (2002), é possível obter de forma comercial extratos de algumas espécies que podem apresentar efeito inseticida, translaminar e sistêmico, acaricida, nematicida, moluscicida, bactericida, fungicida, ou atuando como repelente de insetos. A atuação (contato, ingestão e absorção) e controle de determinadas pragas é dependente da espécie vegetal da qual foi obtido e, em alguns casos, possuem compatibilidade com substâncias químicas como organofosforados e carbamatos, permitindo sua utilização em projetos de MIP (Manejo Integrado de Pragas).

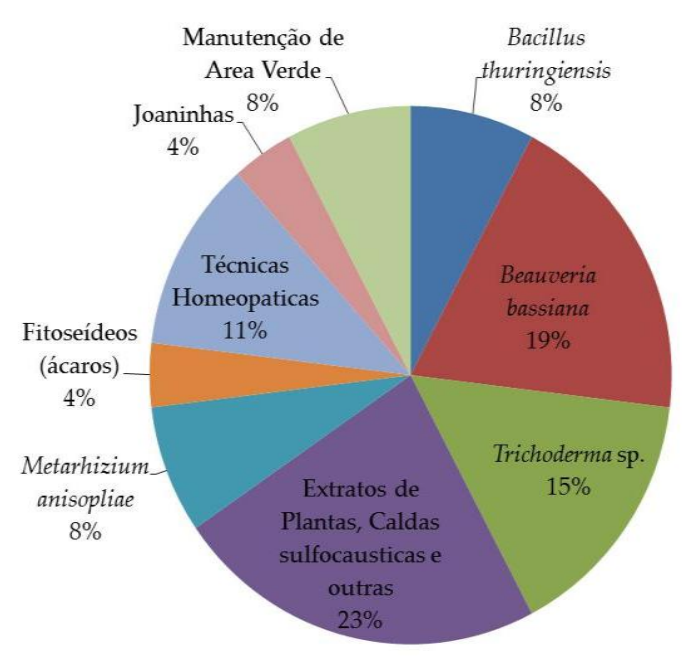

Figura 3 - Técnicas de biocontrole e de manutenção da biodiversidade aplicada pelos agricultores da cidade de Maringá-PR

Alguns trabalhos desenvolvidos com extrato bruto ou óleo essencial de plantas medicinais da flora nativa indicam o potencial das mesmas no controle de fitopatógenos, por sua ação fungitóxica direta, inibindo o crescimento micelial e a germinação de esporos, quanto pela indução de fitoalexinas, que são metabólitos secundários antimicrobianos, produzidos pelas plantas em resposta a estresses físicos, químicos ou biológicos, 
capazes de inibir ou reduzir a atividade de agentes patogênicos. Tais compostos com características de elicitores têm sido utilizados para estudos, in vitro, de inibição de crescimento micelial e esporulação de fungos fitopatogênicos (MOTOYAMA et al., 2003; STANGARLIN et al., 2010; PURKAYASTHA, 1995; SCHAWANESTRADA; STANGAR-LIN; CRUZ, 2000).

Como agentes de biocontrole, os fungos se destacam entre os microrganismos entomopatogênicos (BARROS et al., 2010), sua produção no Brasil é realizada principalmente com uma mistura triturada contendo arroz e fungo, e comercializada na forma de pómolhável (FARIA; MAGALHÃES, 2001).

Apresentam vantagem por seu mecanismo especializado de infecção, que ocorre com sua penetração ativa nos hospedeiros, não dependendo, da sua ingestão para que o processo infeccioso seja iniciado (FRANCESCHINI et al., 2001).

Os principais fungos entomopatogênicos utilizados no setor agrícola brasileiro são Metarhizium anisopliae (Metschn.) Sorokīn (BARROS et al., 2010) e Beauveria bassiana (Bals.Criv.) Vuill., que possuem atuações no controle de pragas diversas em cultivo de mamão, café, citrus e horticultura (FARIA; MAGALHÃES, 2001), e o micoparasita Trichoderma sp., que pode atuar no controle de fungos fitopatogênicos (BENÍTEZ et al., 2004).

Para cultivo de vegetais, milho, frutas, cereais, orquídeas, silvicultura e algodão são indicados controles com bactérias como a Bacillus thuringiensis
(Bacillales: Bacillaceae), sua eficácia está relacionada em sua capacidade de produzir substâncias entomopatógenas (MORA, 2002).

Com o objetivo de revitalizar o cultivo, podem-se utilizar métodos homeopáticos, que envolvem a aplicação de produtos à base de plantas medicinais ou de minerais, tornando as plantas suficientemente estruturadas, para expressar todo o seu potencial genético e estar em condições de superar os antagonismos ambientais aos quais estão expostas (CÂMARA, 2009).

Segundo Rossi et al. (2004) bioterápicos vem sendo produzidos a partir de insetos e patógenos que atacam as plantas, com o objetivo de induzir resistência. Semelhantemente é possível realizá-la utilizando vegetais com o objetivo de equilibrar seu desenvolvimento no ambiente de cultivo

A preocupação com o impacto da agricultura no meio ambiente é crescente e vários trabalhos são realizados acerca da seleção e eficácia de controladores naturais (Tabela 1). $\mathrm{O}$ uso intensivo de agrotóxicos no controle de doenças, pragas e plantas daninhas na agricultura tem promovido diversos problemas de contaminação ambiental e dos alimentos, além da resistência de tais organismos. Os presentes aspectos impulsionam mudanças nos hábitos de produção, destacando aos alimentos de sistemas de produção orgânica um alto valor pelos benefícios agregados.

Porém, o potencial dessas técnicas ainda se limita pelo baixo incentivo a pesquisas e desenvolvimento de técnicas, aliadas ao desconhecimento sobre o tema. 
Tabela 1 - Alguns trabalhos apresentando o controle biológico natural ou aplicado para pragas citadas por agricultores do sistema orgânico de produção da cidade de Maringá (Existem variações quanto a métodos, épocas e locais de aplicação)

\begin{tabular}{|c|c|c|}
\hline PRAGAS & ESPÉCIES & $\begin{array}{l}\text { ESTUDOS SOBRE POSSÍVEIS } \\
\text { CONTROLADORES }\end{array}$ \\
\hline \multirow{4}{*}{$\begin{array}{l}\text { Antracnose } \\
\text { (Colletotrichum } \\
\text { sp.) }\end{array}$} & $\begin{array}{l}\text { Colletotrichum lagenarium (Berk. \& } \\
\text { Mont.) Arx. (Ascomycota); } \\
\text { Colletotrichum sp. (Ascomycota); }\end{array}$ & $\begin{array}{l}\text { Trichoderma spp. (Ascomycota) (BENÍTEZ et al., 2004; } \\
\text { SILVA et al., 2011). }\end{array}$ \\
\hline & $\begin{array}{l}\text { Colletotrichum musae (Berk. \& M.A. } \\
\text { Curtis) Arx. (Ascomycota); }\end{array}$ & $\begin{array}{l}\text { Óleo de soja, óleo vegetal (pós colheita) (JUNQUEIRA et } \\
\text { al., 2003b). }\end{array}$ \\
\hline & $\begin{array}{l}\text { Colletotrichum gloeosporioides (Penz.) } \\
\text { Penz. \& Sacc. (Ascomycota); }\end{array}$ & Óleo de soja (pós colheita) (JUNQUEIRA et al., 2003a). \\
\hline & $\begin{array}{l}\text { Colletotrichum graminicola Ces.) } \\
\text { G.W. Wilson. (Ascomycota); }\end{array}$ & $\begin{array}{l}\text { "Carqueja" - Baccharis trimera (Less) DC (Magnoliophyta) } \\
\text { in vitro (SCHAWAN-ESTRADA; STANGAR-LIN; CRUZ, } \\
\text { 2000). }\end{array}$ \\
\hline \multirow[b]{2}{*}{ Cupins } & $\begin{array}{l}\text { Nasutitermes coxipoensis Holmgren } \\
\text { (Arthropoda); }\end{array}$ & $\begin{array}{l}\text { Metarhizium anisopliae var. anisopliae (Metschn.) Sorokīn } \\
\text { (Ascomycota) (ALBUQUERQUE et al., 2005). }\end{array}$ \\
\hline & $\begin{array}{l}\text { Cornitermes cumulans Kollar } \\
\text { (Arthropoda); }\end{array}$ & $\begin{array}{l}\text { Beauveria bassiana (Bals.-Criv.) Vuill. (Ascomycota) e } \\
\text { Metarhizium anisopliae (Metschn.) Sorokīn (Ascomycota) } \\
\text { (TOSCANO et al., 2010). }\end{array}$ \\
\hline Formigas & $\begin{array}{l}\text { Atta sexdens rubropilosa Forel } \\
\text { (Arthropoda); }\end{array}$ & $\begin{array}{l}\text { Sesamum indicum L. (Magnoliophyta) (iscas com farinha } \\
\text { de folha de gergelim 15\%) (FILHO; DORVAL, 2003). }\end{array}$ \\
\hline \multirow[b]{2}{*}{ Pulgões } & $\begin{array}{l}\text { Cinara atlantica Wilson } \\
\text { (Arthropoda); }\end{array}$ & $\begin{array}{l}\text { Hippodamia convergens Guérin-Méneville (Arthropoda), } \\
\text { Cycloneda sanguinea L. (Arthropoda) (OLIVEIRA; } \\
\text { WILCKEN; MATOS, 2004); }\end{array}$ \\
\hline & $\begin{array}{l}\text { Rhopalosi- phum padi L. } \\
\text { (Arthropoda); } \\
\text { Sitobion avenae Fabricius } \\
\text { (Arthropoda); } \\
\text { R. phum maidis Fitch (Arthropoda); } \\
\text { Metopophium dirhodum Walker } \\
\text { (Arthropoda); }\end{array}$ & $\begin{array}{l}\text { Lysiphlebus testaceipes Cresson (Arthropoda), } \\
\text { Diaretiella rapae McIntosh (Arthropoda) (ALVES et al., } \\
\text { 2005). }\end{array}$ \\
\hline Percevejos & $\begin{array}{l}\text { Euschistus heros Fabricius } \\
\text { (Arthropoda); }\end{array}$ & $\begin{array}{l}\text { Telenomus podisi Ashmead (Artropoda) - } \\
\text { parasita(LAUMANN et al., 2009). }\end{array}$ \\
\hline Mosca-Branca & $\begin{array}{l}\text { Bemisia tabaci Gennadius } \\
\text { (Arthropoda); }\end{array}$ & $\begin{array}{l}\text { Óleo de Neem (efetivo quando em fase de ninfas) } \\
\text { (MULLA; SU, 1999). } \\
\text { Fungos Verticillium lecanii (Zimm.) Viégas (Ascomycota), } \\
\text { Paecilomyces fumosoroseus (Wize) A.H.S. Br. \& G. Sm. } \\
\text { (Ascomycota), Beauveria bassiana (Bals.-Criv.) Vuill. } \\
\text { (Ascomycota) (FARIA; WRAIGHT, 2001). }\end{array}$ \\
\hline $\begin{array}{l}\text { Vaquinha } \\
\text { (Patriota) }\end{array}$ & Diabrotica sp. (Arthropoda); & $\begin{array}{l}\text { Óleo e/ou pó de Cinamomo (Melia azedarach L. } \\
\text { (Magnoliophyta)) (SOUZA et al., 2015). }\end{array}$ \\
\hline Lagartas & $\begin{array}{l}\text { Helicoverpa armigera Hubner } \\
\text { (Arthropoda); }\end{array}$ & $\begin{array}{l}\text { Fungo Nomuraea rileyi (Farl.) Samson } 1974 \text { (Ascomycota) } \\
\text { (COSTA et al., 2015). } \\
\text { Fungo Penicillium ochrochloron Biourge (Ascomycota) } \\
\text { (PATIL; JADHAV, 2015). }\end{array}$ \\
\hline \multirow[t]{2}{*}{ Ácaros } & - & $\begin{array}{l}\text { Beauveria bassiana (Bals.-Criv.) Vuill. (Ascomycota) e } \\
\text { Metarhizium anisopliae (Metschn.) Sorokīn (Ascomycota) } \\
\text { (SHI; ZHANG; FENG, 2008). }\end{array}$ \\
\hline & $\begin{array}{l}\text { Oligonychus ilicis McGregor } \\
\text { (Arthropoda); }\end{array}$ & Euseius alatus DeLeon (Arthropoda) (TOLEDO, 2015). \\
\hline
\end{tabular}


Segundo Parra (2014), os maiores desafios para a aplicação de biocontroladores no Brasil são: 1Informar sobre o uso e vantagens da utilização do biocontrole; 2Necessidade de maiores atividades de extensão, ou seja, transferência de tecnologia universidade-empresacampo; 3- Desenvolvimento de técnicas para o monitoramento de pragas em pequenas e grandes áreas; 4Disponibilidade de produtos de biocontrole para atender a demanda da grande área rural brasileira; 5Qualidade destes produtos; 6- Logística (armazenamento e transporte); 7- A falta de uma Legislação adequada para a comercialização de inimigos naturais (a legislação atual é uma adaptação dos pesticidas químicos); 8- Seletividade química dos agrotóxicos para integrar os inimigos naturais dentro do contexto do MIP; 9- O desenvolvimento de técnicas eficazes de aplicação dos biocontroladores; 10- A dificuldade de adaptação ao dinamismo da agricultura brasileira, considerando a alta variação climática e entomológica no decorrer do ano ou época de plantio de certas cultivares; 11- A limitação de tecnologias para plantas transgênicas, tais como as que utilizam a tecnologia $\mathrm{Bt}$, que controlam pragas como lepidópteros e coleópteros, mas não os insetos sugadores, o que acaba por causar um desequilíbrio na entomofauna, podendo afetar plantas e áreas vizinhas.

É necessário considerar os benefícios ecológicos advindos do uso de controle biológico. Assim, tais técnicas devem ser exploradas como uma alternativa importante na redução
(MIP) ou total eliminação do uso de agrotóxicos.

\section{Conclusões}

A utilização de biocontroladores é considerada uma alternativa viável entre os produtores entrevistados, com resultados satisfatórios para um sistema de produção orgânica, substituindo defensivos químicos e proporcionando a manutenção do equilíbrio ambiental.

São necessários futuros estudos relacionados ao levantamento da entomofauna e bioprospecção de controladores principalmente voltados para as culturas de interesse como as hortaliças.

Trabalhos de extensão universitária envolvendo empresas e os agricultores são importantes para a ampliação do sistema orgânico de produção.

\section{Agradecimentos}

A EMATER (Empresa Brasileira de Extensão Rural, unidade MaringáPR) pelos dados fornecidos e aos agricultores da POMAR (Associação dos Produtores Orgânicos de Maringá) pela participação.

\section{Referências}

ALBUQUERQUE, A. C. et al. Patogenicidade de Metarhizium anisopliae var. anisopliae e Metarhizium anisopliae var. acridum sobre Nasutitermes coxipoensis (Holmgren) (Isoptera: Termitidae). Neotrop. Entomol., v. 34, n. 4, 2005. 
ALVES, L. F. A. et al. Controle biológico natural de pulgões (Hemiptera: Aphididae) em lavoura de trigo por parasitóides (Hymenoptera, Aphidiinae), no município de Medianeira, PR, Brasil. Semina: Ciências Agrárias, v. 26, n. 2, p. 155-160, 2005.

BARROS, N. M. et al. Fungos como agentes de controle de pragas. In: ESPOSITO, E.; AZEVEDO, J. L. (Org.). Fungos: uma introdução à biologia, bioquímica e biotecnologia. 2. ed. Caxias do Sul: Educs, 2010. p. 491-532.

BENÍTEZ, T. et al. Biocontrol mechanisms of Trichoderma strains. International microbiology, v. 7, p. 249-260, 2004.

CÂMARA, F. L. A. Controle de pragas com homeopatia. Hortic. bras., v. 27, n. 2, 2009. Disponível em: $<$ http://www.abhorticultura.com.br/even tosx/trabalhos/ev 3/P 26 Artigo Francis co Ara\%C3\%BAjo C\%C3\%A2mara.pdf> . Acesso em: 20 Jul. 2015.

CHAGAS, A. C. S. Controle de parasitas utilizando extratos vegetais. Rev. Bras. Parasitol. Vet., v. 13, p. 156-160, 2004.

COSTA, V. H. D. et al. Nomuraea rileyi (Hypocreales: Clavicipitaceae) in Helicoverpa armigera (Lepidoptera: Noctuidae) larvae in Brazil. Florida Entomologist, v. 98, n. 2, p. 796-798, 2015.

DEBACH, P.; ROSEN, D. Biological Control by Natural Enemies. 2. ed. Cambridge: Cambridge University Press, 1991. $386 \mathrm{p}$.

FARIA, M. R.; MAGALHÃES, B. P. O uso de fungos entomopatogênicos no Brasil: Situação atual e perspectivas. Biotecnologia Ciência \& Desenvolvimento, n. 22, p. 18-21, 2001.
FARIA, M.; WRAIGHT, S. P. Biological control of Bemisia tabaci with fungi. Crop protection, v. 20, n. 9, p. 767-778, 2001.

FILHO, O. P.; DORVAL, A. Efeito de formulações granuladas de diferentes produtos químicos e à base de folhas e de sementes de gergelim, Sesamum indicum, no controle de formigueiros de Atta sexdens rubropilosa Forel, 1908 (Hymenoptera: Formicidae). Ciência Florestal, v. 13, n. 2, p. 67-70, 2003.

FRANCESCHINI, M. Biotecnologia aplicada ao controle biológico. Biotecnologia, ciência e desenvolvimento, n. 23, p. 32-37, 2001.

GALLO, D. et al. Entomologia Agrícola. 1. ed. Piracicaba: FEALQ, 2002. 920 p.

JUNQUEIRA, N. T. V. et al. Efeito de produtos biológicos e químicos no controle da antracnose e na conservação da manga cv. Palmer em pós-colheita. Planaltina, DF: Embrapa Cerrados, 2003a. 14 p.

JUNQUEIRA, N. T. V. et al. Potencial de defensivos biológicos no controle da antracnose e na conservação de bananas na pós-colheita. Planaltina, DF: EMBRAPA CERRADOS. 14 p. 2003b.

LACEY, L. A. et al. Insect Pathogens as Biological Control Agents: Do They Have a Future?. Biological Control, v. 21, p. 230-248, 2001.

LAUMANN, R. A. et al. Response of the egg parasitoids Trissolcus basalis and Telenomus podisi to compounds from defensive secretions of stink bugs. Journal of chemical ecology, v. 35, n. 1, p. 8-19, 2009. 
LIMA, M. C.; ROCHA, S. A. Efeitos dos agrotóxicos sobre as abelhas silvestres no brasil. Brasília: IBAMA, 2012. 88 p. ISBN 978-85-7300-366-6.

MORA, J. D. Bioplaguicidas: Guía de ingredientes activos em América Central. Costa rica: CATIE, 2002. 153 p. ISBN $997757382-4$.

MORAIS, L. A. S. Óleos essenciais no controle fitossanitário. In: BETTIOL, W.;

MORANDI, M. A. B. Biocontrole de doenças de plantas: Uso e perspectivas. 1. ed. Jaguariúna: EMPRAPA, 2009. p. 139-152.

MOTOYAMA, M. M. et al. Indução de fitoalexinas em soja e em sorgo e efeito fungitóxico de extratos cítricos sobre Colletotrichum lagenarium e Fusarium semitectum. Acta Scientiarum Agronomy, v. 25, n. 2, p. 491-496. 2003.

MULLA, M. S.; SU, T. Activity and biological effects of neem products against arthropods of medical and veterinary importance. Journal of the American Mosquito Control Association, v. 15, n. 2, p. 133-152, 1999.

OLIVEIRA, N. C.; WILCKEN, C. F.; MATOS, C. A. O. Ciclo biológico e predação de três espécies de coccinelídeos (Coleoptera, Coccinellidae) sobre o pulgão-gigante-do-pinus Cinara atlantica (Wilson) (Hemiptera, Aphididae). Rev. Bras. entomol.,v. 48, n. 4, 2004.

PARRA, J. R. P. Situação atual e perspectivas do controle biológico, através de liberações inundativas no Brasil. Pesq. agropec. bras., v. 27, p. 271279, 1992.
PARRA, J. R. P. Biological Control in Brazil: an overview. Sci. agric, v. 71 , n. 5, p. 420429, 2014.

PATIL, N. S.; JADHAV, J. P. Significance of Penicillium ochrochloron chitinase as a biocontrol agent against pest Helicoverpa armigera. Chemosphere, v. 128 , p. 231235, 2015.

PURKAYASTHA, R. P. Progress in phytoalexin research during the past 50 years. In: DANIEL, M.; PURKAYASTHA, R. P. (Ed.). Handbook of phytoalexin metabolism and action. New York: Marcel Dekker, 1995. p. 1-39.

ROSSI, F. et al. Emprego da homeopatia no controle de doenças de plantas. Summa Phytopathologica, v. 30, n. 1, p. 156-158, 2004.

SCHAWAN-ESTRADA, K. R. F.; STANGARLIN, J. R.; CRUZ, M. E. S. Uso de extratos vegetais no controle de fungos fitopatogênicos. Revista Floresta, v. 30, p. 129-137, 2000.

SHI, W.B.; ZHANG, L.L.; FENG, M.G. Field trials of four formulations of Beauveria bassiana and Metarhizium anisoplae for control of cotton spider mites (Acari: Tetranychidae) in the Tarim Basin of China. Biological control, v. 45,n. 1, p. 48-55, 2008.

SILVA, M. B. et al. Extratos de plantas e seus derivados no controle de doenças e pragas. In: VENZON, M.; JÚNIOR, T. J. P.; PALLINI, A. Controle alternativo de pragas e doenças em agricultura orgânica. 1. ed. Viçosa: EPAMIG, 2010. p. 33-54.

SILVA, V. N. et al. Promoção de crescimento e indução de resistência à antracnose por Trichoderma spp. em 
pepineiro. Pesq. agropec. bras., v. 46, n. 12, p. 1609-1618, 2011.

SOUZA, B. H. S. et al. Formulations of Melia azedarach to control Diabrotica speciosa (Germar) (Coleoptera: Chrysomelidae) larvae in corn and plant enhancement. Neotropical entomology, v. 44, n. 2, p. 173-179, 2015.

STANGARLIN, J. R. et al. Indução de fitoalexinas em soja e sorgo por preparações de Saccharomyces boulardii. Arq. Inst. Biol, v. 77, n. 1, p. 9198, 2010.

TOLEDO, M. A. Estudo de métodos não convencionais visando o controle do ácaro Oligony chusilicis (McGregor, 1917) (Acari: Tetranychidae) em cafeeiro (Coffea arabica L.). 2015.

TOSCANO, L. C. et al. Controle do cupim de montículo (isoptera: termitidae) de pastagem com fungos entomopatogênicos. Revista Caatinga, v. 23, n. 2, p. 6-11, 2010.

VAN DRIESCHE, R. G. et al. Classical biological control for the protection of natural ecosystems. Biological Control, v. 54, n. 11, p. S2-S33, 2010. 